\title{
Isokinetic Performance of Shoulder External and Internal Rotators in Adolescent Male Volleyball Athletes \\ ORIGINAL
}

Kelly Cristine Franceschini ${ }^{1}$, Naíma Nissola1,

Bruno Soldatelli Zardo', Gerson Sacilotto Tadielo², Leandro Viçosa Bonetti ${ }^{3}$

1 Physical Therapy Student, Universidade de Caxias do Sul, Rio Grande do Sul, Brazil.

\section{Abstract}

Background: Volleyball is a sport characterized by short, intensive and explosive actions. In the performance of these repetitive movements, the shoulder joint receives the largest load. Therefore, a detailed assessment of biomechanics and muscle performance is fundamental in the evaluation, treatment and in prevention of shoulder injuries in volleyball athletes.

Methods and Findings: Information from a database concerning the isokinetic evaluation of the shoulder's external and internal rotator muscles at the $60 \% \mathrm{~s}$ and $180 \% \mathrm{~s}$ angular velocities from 20 adolescent male volleyball athletes under 17 years of age was assessed. At a velocity of $60 \% \mathrm{~s}$ the average values for internal rotators on the nondominant limb were significantly smaller than those of the dominant limb, and at a velocity of $180 \%$ both the internal and external rotators average values for the non-dominant limb were significantly smaller than those for the dominant limb. For the external-internal rotator ratio analyses, there was no significant difference between the limbs.

Conclusions: In conclusion, the unilateral demands during typical movements in volleyball resulted in higher peak torque values for the dominant limb in comparison to that of the non-dominant limb. For the external-internal rotators ratio, no relevant differences were found between limbs, and the average values were within the parameters of normality.

\section{Introduction}

Volleyball is a sport characterized by short, intensive and explosive actions such as lateral movements, jumping, serving, passing, setting,

2 PT, MSc, Professor of Physical Therapy, Universidade de Caxias do Sul, Rio Grande do Sul, Brazil.

3 PT, PhD, Professor of Physical Therapy, Universidade de Caxias do Sul, Rio Grande do Sul, Brazil

\section{Contact information:}

Leandro Viçosa Bonetti,

PT, PhD, Professor of Physical Therapy.

Address: Universidade de Caxias do Sul, RS, Brazil. Rua Francisco Getúlio Vargas, 1130, Bloco 70, Caxias do Sul, RS, Brasil, CEP 95070-560.

” leandrovbonetti@gmail.com Ivbonetti@ucs.br

\section{Keywords}

Sports; Muscle Strength;

Torque. 
blocking, digging, diving and spiking [1]. The most important actions observed during a match are the offensive actions -serve, block and spike- that demand repeated movements and intense articular and muscular performance [2-3]. The shoulder joint receives the largest load during repetitive volleyball movements and consequently many injuries affect this joint during practicing volleyball [4]. The shoulder is responsible for $16 \%$ of injuries in elite volleyball players [5] and a recent study with world-class volleyball athletes showed $20.7 \%$ of these injuries were related to overuse [6].

A set of mono, bi and triarticular muscles maintain stability and coordinated shoulder movements [7]. Nevertheless, the usual movements performed by volleyball athletes seem to predispose them to a muscular imbalance between external and internal rotators [8], the identification of which is important to prevent overuse shoulder injuries [5]. However, a detailed assessment of biomechanics and muscle performance is fundamental in the evaluation, treatment and prevention of shoulder injuries in players. At present there is a lack of studies related to the evaluation of isokinetic parameters which could reveal important information about possible risk factors for shoulder injuries in volleyball players [9]. The well-established outcome parameter in isokinetic analyses is peak torque (PT) [10], and considering volleyball asymmetric movements of the shoulder, imbalances in PT values between the dominant (D) and non-dominant (ND) shoulders are expected. Thus, the main objective of the present study was to analyze, using information from a database, the muscular performance and the unilateral differences and relationships between the shoulders' external and internal rotators in adolescent male volleyball athletes.

\section{Methods}

This is a quantitative, cross-sectional and retrospective study conducted at the Instituto de Me- dicina do Esporte e Ciências Aplicadas ao Movimento Humano da Universidade de Caxias do Sul (IME-UCS), in the city of Caxias do Sul, Rio Grande do Sul, Brazil. It has been approved (protocol number 967.527) by the Ethical Research Committee of the Faculdade Cenecista Bento Gonçalves (Bento Gonçalves, Rio Grande do Sul, Brazil), and conducted according to the 2012 Law N466 of the National Health Council, that approves the guidelines and rules for research involving human beings. The information provided by the SMI-UCS database concerning the isokinetic evaluation of the shoulder external and internal rotator muscles from 20 male volleyball athletes under 17 years of age from the Universidade de Caxias do Sul team are part of this study's sample. Such a number was conveniently established and, therefore determined intentionally and not by probability, according to the number of available evaluations on the IME-UCS' database. These evaluations were made with the institution's isokinetic dynamometer (Biodex System 4®, Biodex Medical Systems, Shieley, New York, USA). The chosen samples were made between November and December 2014, in a concentric-concentric mode, for the shoulder's external and internal rotator muscles at the angular velocities of $60 \% \mathrm{~s}$ and $180 \% \mathrm{~s}$. The evaluations in which the respective IME-UCS' consent term had not been authorized by the athlete were excluded from this study. The collected evaluations were performed according to a standardized protocol, whereby a questionnaire concerning age, height, weight, category, dominance, field position, and prevalence of injuries, among other aspects, was presented to the athletes who in sequence, underwent warm-up exercises on a stationary bicycle at moderate velocity for 8 minutes. The athletes were then conducted through the isokinetic dynamometer. Athletes sat in the dynamometer chair with their torso leaning at $85^{\circ}$, and they were stabilized by means of belts around the torso and pelvis to avoid compensatory movements. The po- 
sitioning of the superior limb to assess the shoulders' external and internal rotators was as follows: $60^{\circ}$ shoulder abduction in the scapular plane $\left(30^{\circ}\right.$ ahead of the frontal plane) and $90^{\circ}$ elbow flexion; the wrist was stabilized to avoid substitution of movement. The amplitude of the movement was limited at $40^{\circ}$ external rotation and $50^{\circ}$ internal. Tests were conducted first with the dominant limb (DL) and second with the non-dominant limb (NDL). The athletes performed three submaximal repetitions and a previous maximal to each test at all four velocities to familiarize themselves with the procedures and to warm up. Protocol during the test demanded 5 maximal repetitions of internal and external shoulder rotation in concentric-concentric mode, at the angular velocity of $60 \%$, and 10 repetitions at the angular velocity of $180 \%$ s. A one-minute rest period was given between the evaluations of different velocities, and a threeminute rest period between the DL and NDL evaluations. Athletes were tested along with the use of verbal incentives to stimulate them throughout the process, and encourage the maximum use of their strength potential. Isokinetic variables -peak torque $(\mathrm{PT}, \mathrm{N} / \mathrm{m})$ and the external-internal rotator ratio (ER/IR ratio- were used in the analysis. Initially, anthropometric data (weight, height and body mass index) underwent descriptive analysis. The mean values for PT and ER/IR ratio for shoulder musculature were evaluated statistically using SPSS 17.0 software (Statistical Package to Social Science for Windows). To verify the normality of the data distribution, the Shapiro-Wilk test was used, and the mean values for the DL and NDL evaluations were submitted to Student's t-test, with a resultant level of significance of 0.05 .

\section{Results}

We accessed isokinetic evaluations from 20 adolescent male volleyball athletes from the Universidade de Caxias do Sul team. The athletes' mean age was 15.95 ( \pm 0.78 ) years old, mean height was $1.85( \pm$ 0.09) meters, mean weight was 76.05 ( \pm 10.91) kilograms and the mean body mass index test presented an average result of $24.57( \pm 2.58) \mathrm{kg} / \mathrm{m}^{2}$, considered normal [11]. Among the tested athletes, 18 reported a dominance of the right limb for game movements and only 2 reported dominance of the left limb. Out of the total, 16 players had not presents injuries during the previous 45 days to the isokinetic evaluation, while 4 had suffered injuries both on practice and competition, one of them reported a traumatic injury due to glenohumeral sprain and three others reported non-traumatic injuries with clinical diagnosis of tendinitis. None of these 4 athletes needed surgical procedure or to keep away from practice, and only one of them underwent physical therapy for a month. None of the athletes reported symptoms or complaints of pain at the moment of the isokinetic evaluation.

With regard to the isokinetic data results, the average values of PT for the DL and NDL are presented on Table 1. At the velocity of $60 \% \mathrm{~s}$ the average values for IR on the NDL was significantly smaller than those of the DL and at the velocity of $180 \% \mathrm{~s}$, both the IR and ER average values on the NDL were significantly smaller than those of the DL.

Table 1. Mean and standard deviation values for peak torque of the external and internal rotators musculatures of the dominant limb and the non-dominant limb's shoulder.

\begin{tabular}{|c|c|c|c|c|c|c|}
\hline \multirow{2}{*}{$\begin{array}{l}\text { Angular } \\
\text { Velocities }\end{array}$} & \multicolumn{3}{|c|}{ PT External Rotators (N/m) } & \multicolumn{3}{|c|}{ PT Internal Rotators (N/m) } \\
\hline & DL & NDL & $p$ & $\mathrm{DL}$ & NDL & $p$ \\
\hline $60 \% / 5$ & $35.48( \pm 9.63)$ & $32.93( \pm 7.28)$ & 0.101 & $57.05( \pm 10.73)$ & $50.04( \pm 9.06)^{*}$ & 0.005 \\
\hline $180 \% / \mathrm{s}$ & $36.17( \pm 9.44)$ & $33.91( \pm 7.86)^{*}$ & 0.003 & $51.45( \pm 10.09)$ & $45.72( \pm 9.90)^{*}$ & 0.014 \\
\hline
\end{tabular}


Table 2. Mean and standard deviation values for external-internal rotators ratio (ER/IR ratio) of the dominant limb and the non-dominant limb's shoulder.

\begin{tabular}{|c|c|c|c|}
\hline \multirow{2}{*}{$\begin{array}{l}\text { Angular } \\
\text { Velocities }\end{array}$} & \multicolumn{3}{|c|}{ ER/IR ratio (\%) } \\
\hline & $\mathrm{DL}$ & NDL & $p$ \\
\hline $60 \% / 5$ & $62.00( \pm 10.25)$ & $66.34( \pm 11.37)$ & 0.177 \\
\hline $180 \% / \mathrm{s}$ & $70.65( \pm 12.08)$ & $74.09( \pm 13.70)$ & 0.203 \\
\hline
\end{tabular}

Table 2 showed that there was no significant difference between $\mathrm{DL}$ and NDL results of the ER/IR for the angular velocities of $60 \% \mathrm{~s}$ and $180 \% \mathrm{~s}$. It also shows that the ER/IR ratio for the NDL is higher than that for the DL on both velocities assessed.

\section{Discussion}

Isokinetic evaluation is extensively used for studying shoulder muscle performance in healthy athletes [12] and asymmetrical actions that lead to a greater risk of injury to the dominant shoulder [13]. Volleyball is characterized by highly asymmetric kinematic patterns in its basic skills, especially with respect to the upper limbs, causing overload and consequent muscular imbalance [8]. However, the present study aimed to analyze isokinetic performance of the shoulders' external and internal rotators of adolescent male volleyball players. Regarding shoulder injuries, our results showed that only $20 \%$ of the athletes had presented with some kind of shoulder injury related to volleyball practice. Reeser et al. [13] reported that $57 \%$ of male volleyball athletes (21.5 years average age) informed them of a history of shoulder problems during the preceding competitive season. However, Beneka et al. [14] recorded the injury rate of all age categories of Greek male volleyball and confirmed that the prevalence of injuries for 15-18-year-old volleyball players was significantly lower than that for older players. According to Reeser et al. [10], age, position played, and serving style are three possible factors associated with shoulder problems. Mohseni-Bandpei et al. [4] related that the prevalence of pain is directly related to the years of practice of the sport, frequency of practice and competitive level, with those athletes who practice the sport for over 5 years, at national competition level, practicing over 4 times a week and performing constant repetitive movements, being more prone to shoulder pain. In our study, we found that athletes had practiced for an average of 10.32 hours per week over a period of around 3.5 years-therefore, the inferior values, in comparison to those of Mohseni-Bandpei et al [4], are in accordance with the lower rate of shoulder pains. Seventy-five percent of related shoulder injuries were non-traumatic and associated with overuse injuries. In volleyball practices, previous studies showed that the shoulder and the knee were the most frequently overuse injuries [15]. Volleyball is a sport that demands a lot of the glenohumeral joint; consequently, injuries to this joint are frequent among the sport's practitioners [8].

The isokinetic evaluations showed significant differences between the limbs in PT average analyses. The NDL demonstrated average values significantly smaller than the DL in the IR analyses at $60 \%$ and $180 \%$ s. Such data are also in agreement with Mendonça et al.'s [8] findings, which assessed 35 athletes in the under-19 and under-21- year-old categories for the Brazilian volleyball team and found significant differences at $60 \% \mathrm{~s}$, and in which the DL's strength prevailed over the NDL for both categories. At the same velocity Wang, Macfarlane and Cochrane [16] evaluated 10 athletes from England's national men's volleyball team, Michael et al. [17] evaluated 16 German volleyball players, Markou and Vagenas [18] evaluated 24 elite male offensive players from Greece and Hadzic et al. [9] evaluated 99 Slovenian first and second national divisions. Their results illustrated muscle strength asymmetry in the upper limbs, with the dominant upper extremity tending to produce higher values for PT compared 
to the non-dominant side. At $60 \% \mathrm{~s}$ and $180 \%$, the results of 16 male athletes from the English national volleyball team [19] and from 35 players from the highest division of Dutch volleyball [20], were the same as the results of this study, with the dominant arm having significantly higher PT average values than the non-dominant one. Also Wang, Macfarlane and Cochrane [16] at 120\%, and Harput et al. [21] at $90 \%$, illustrated that limb dominance has an effect on IR muscles; however, Harput et al. [21] showed higher average values for the NDL in 40 adolescent volleyball players' (male and female) evaluations.

During ER analyses, only at the angular velocity of $180 \%$ s were the PT average value of the NDL significantly lower than those of the DL. When comparing this data with other volleyball studies, at the $60 \%$ angular velocity $[8,9,17,18,20]$, and at the $90 \%$ [21], no significant differences were demonstrated between the DL and the NDL with respect to PT average analyses. At the angular velocity of $60 \%$, Wang and Cochrane [19] found significantly different PT averages between the limbs, however, the NDL demonstrated higher values than the DL. At the $180 \%$ analyses, Van Cingel et al. [20] demonstrated no significant differences between limbs in PT average value of the ER, which differs from the results of this study. Nevertheless, in another high angular velocity analysis, Mendonça et al. [8] found the average PT value for the NDL to be significantly lower than that of the DL in the under-19 and under-21 age categories.

These significant differences in PT between the limbs, mainly in IR muscles, confirm the asymmetric volleyball characteristics during upper limb movements. Based on this data, this PT differential in shoulder function of volleyball players can only be attributed to the fact that the dominant side shoulder develops a systematic superiority in overall muscle strength over the years [18]. Furthermore, according to Mendonça et al. [8], such differences are consequence of the gain in IR muscular strength of the DL due to repetitive acceleration movements during the strike. Other asymmetrical sports such as handball [22], tennis [23-24], baseball [25] and water polo [26] demonstrate a preference for the DL. When comparing IR and ER PT average value, IR muscles presented average values higher than the ER muscles at both assessed angular velocities. This is an expected result both for non-athletes [27] and for volleyball athletes [8, 9, 16, 18-21].

Relative to the balance between IR and ER muscles, the ER/IR ratio analyses are important and the first analyzed data indicated no significant differences between the DL and NDL average values at the two angular velocities. Another important aspect is that the average values for the ER/IR ratio were between $60 \%$ and $75 \%$, which are the regular parameters suggested in the literature for males [27]. If we compare the results for these young athletes to Mendonça's [8] results for the under-19 age category at the $60 \%$ angular $(62 \%$ and $64 \%$ for the DL and NDL, respectively), they are similar, and other volleyball studies have also shown values between those that are normal $[9,18]$. However, volleyball athletes under 21 years of age [8] and other adult athletes $[16,19]$ demonstrated NDL values higher than 75\%, while Michael et al. [17] presented higher values for both the DL and NDL. At 180\% our results demonstrated normal values, however, other studies showed values for the NDL higher than 75\% [19-20], thus confirming an imbalance between the $E R$ and IR in this limb. Overhead athletes showed an association between an abnormal ER/IR ratio and the incidence of shoulder injuries [28]. According to Edouard et al. [29], a player with this imbalance has two and a half times more chances of suffering a shoulder injury.

In conclusion, the unilateral demands during typical movements in volleyball resulted in higher peak torque values for the dominant limb in comparison to those for the non-dominant limb in adolescent athletes' analyses. These results are considered normal for volleyball players and are justified by 
a more frequent use of the dominant limb, and consequently, differential strength production. As for the ER/IR ratio, no relevant differences were found between limbs, and the average values are within the parameters of normality, thus showing joint balance between external and internal rotator muscles in both limbs. Although the results obtained in the present study were similar to other studies with volleyball athletes, no studies has been conducted with adolescent's volleyball players. Our results indicated that volleyball athletes with mean age of 16 years are already presenting adaptations to specific sports training with asymmetric repeated movements. These results may help coaches and sports medicine professionals to understand how muscles of the shoulders develop over the course of practicing volleyball and consequently decreases the risk of injury due to imbalances. In addition, future studies should consider isokinetic testing for adolescent athletes. These tests are necessary to confirm our findings; however, the results presented here may contribute to normality parameters associated with the shoulder joints of adolescent volleyball athletes.

\section{References}

1. Mroczek D, Januszkiewicz A, Kawczynski AS, et al. Analysis of male volleyball players' motor activities during a top level match. J Strength Cond Res. 2014; 28(8):2297-305.

2. Marcelino R, Mesquita I, Sampaio J, Moraes JC. Estudo dos indicadores de rendimento em voleibol em função do resultado do set. Rev Bras Educ Fís Esporte. 2010; 24(1):69-78.

3. Costa GC, Ferreira NN, Junqueira G, Afonso J, Mesquita I. Determinants of attack tactics in youth male elite volleyball. Int J Performance Analysis Sport. 2011; 11(1):96-104.

4. Mohseni-Bandpei MA, Keshavarz R, Minoonejhad $H$, Mohsenifar $\mathrm{H}$, Shakeri $\mathrm{H}$. Shoulder pain in Iranian elite athletes: The prevalence and risk factors. J Manipulative Physiol Ther. 2012; 35(7):541-8.

5. Seminati E, Minetti AE. Overuse in volleyball training/practice: A review on shoulder and spine-related injuries. Eur J Sport Sci. 2013; 13(6):732-43.

6. Bere T, Kruczynski J, Veintimilla N, Hamu Y, Bahr R. Injury risk is low among world-class volleyball players: 4-year data from the FIVB Injury Surveillance System. Br J Sports Med. 2015; 49(17):1132-7.

7. Veegera HEJ, Van Der Helm FCT. Shoulder function: The perfect compromise between mobility and stability. J Biomech. 2007; 40(10):2119-29.

8. Mendonça LDM, Bittencourt NFN, Anjos MTS, Silva AA, Fonseca ST. Avaliação muscular isocinética da articulação do ombro em atletas da seleção brasileira de voleibol sub-19 e sub21 masculino. Rev Bras Med Esporte. 2010; 16(2):107-11.

9. Hadzic V, Sattler T, Veselko M, Markovic G, Dervisevic E. Strength asymmetry of the shoulders in elite volleyball players. J Athl Train. 2014; 49(3):338-44.

10. Dvir Z. Relevant, less relevant and irrelevant isokinetic strength test parameters: Some critical comments. Mov Sport Sci/Sci Mot. 2014; 85(1):15-21.

11. World Healthy Organization [Internet]. Global Health Observatory data repository. Available from: http://apps.who. int/gho/data/node.main. A897A?lang=en.

12. Stickley CD, Hetzler RK, Freemyer BG, et al. Isokinetic peak torque ratios and shoulder injury history in adolescent female volleyball athletes. J Athl Train. 2008; 43(6):571-7.

13. Reeser JC, Joy EA, Porucznik CA, Berg RL, Colliver EB, Willick SE. Risk factors for volleyball-related shoulder pain and dysfunction. PM R. 2010; 2(1):27-36.

14. Beneka A, Malliou P, Gioftsidou A, Tsigganos G, Zetou H, Godolias $G$. Injury incidence rate, severity and diagnosis in male volleyball players. Sport Sci Health. 2009; 5(1):93-9.

15. Verhagen EALM, Van Der Beek AJ, Bouter LM, Bahr RM, Van Mechelen W. A one season prospective cohort study of volleyball injuries. Br J Sports Med. 2004; 38(1):477-81. 
16. Wang $H$, Macfarlane $A$, Cochrane $T$. Isokinetic performance and shoulder mobility in elite volleyball athletes from the United Kingdom. Br J Sports Med. 2000; 34(1):39-43.

17. Michael J, Konig D, Hessling U, Popken F, Eysel P. Results of shoulder isokinetic testing in volleyball players. Sportverletz Sportschaden. 2003; 17(2):71-4.

18. Markou S, Vagenas $G$. Multivariate isokinetic asymmetry of the knee and shoulder in elite volleyball players. Eur J Sport Sci. 2006; 6(1):71-80

19. Wang $H$, Cochrane $T$. Mobility impairment, muscle imbalance, muscle weakness, scapular asymmetry and shoulder injury in elite volleyball athletes. J Sports Med Phys Fitness. 2001; 41(3):403-10.

20. Van Cingel R, Kleinrensink G, Stoeckart R, Aufdemkampe G, de Bie R, Kuipers $H$. Strength values of shoulder internal and external rotators in elite volleyball players. J Sport Rehabil. 2006; 15(1):237-45

21. Harput G, Guney H, Colakoglu FF, Baltac G. Isokinetic strength profile of shoulder internal and external rotators of adolescent volleyball players. Orthop J Sports Med. 2014; 2(3 Suppl).

22. Andrade $M$ dos $S$, de Lira $C A$, Vancini $R L$, de Almeida $A A$, Benedito-Silva AA, da Silva AC. Profiling the isokinetic shoulder rotator muscle strength in 13- to 36-year-old male and female handball players. Phys Ther Sport. 2013; 14(4):246-52.

23. Gozlan G, Bensoussan L, Coudreuse JM, Fondarai J, Gremeaux V, Viton JM, Delarque A. Isokinetic dynamometer measurement of shoulder rotational strength in healthy elite athletes (swimming, volley-ball, tennis): Comparison between dominant and nondominant shoulder. Ann Readapt Med Phys. 2006; 49(1):8-15

24. Saccol MF, Gracitelli GC, da Silva RT, Laurino CF, Fleury AM, Andrade $\mathrm{M}$ dos $\mathrm{S}$, da Silva AC. Shoulder functional ratio in elite junior tennis players. Phys Ther Sport. 2010; 11(1):8-11.

25. Noffal GJ. Isokinetic eccentric-to-concentric strength ratios of the shoulder rotator muscles in throwers and nonthrowers. Am J Sports Med. 2003; 31(4):537-41.
26. Campos TF, Petrone KCO, Navega MT, Renner AF, MattielloRosa SM. Estudo dos picos de torque concêntricos e excêntricos dos rotadores mediais e laterais do ombro de atletas do pólo aquático. Rev Bras Fisioter. 2005; 9(2):137-43.

27. Ellenbecker TS, Davies GJ. The application of isokinetics in testing and rehabilitation of the shoulder complex. J Athl Train. 2000; 35(3):338-50.

28. Byram IR, Bushnell BD, Dugger K, Charron K, Harrell FE Jr, Noonan TJ. Preseason shoulder strength measurements in professional baseball pitchers: Identifying players at risk for injury. Am J Sports Med. 2010; 38(7):1375-82.

29. Edouard P, Degache F, Oullion R, Plessis JY, Gleizes-Cervera $S$, Calmels P. Shoulder strength imbalances as injury risk in handball. Int J Sports Med. 2013; 34(7):654-60.
Publish in International Archives of Medicine

International Archives of Medicine is an open access journal publishing articles encompassing all aspects of medical science and clinical practice. IAM is considered a megajournal with independent sections on all areas of medicine. IAM is a really international journal with authors and board members from all around the world. The journal is widely indexed and classified Q1 in category Medicine. 